\title{
ATIVIDADES DE ENFERMAGEM QUE CONTRIBUEM PARA A QUALIDADE DOS CUIDADOS: ANÁLISE FATORIAL CONFIRMATÓRIA DA ESCALA
}

\author{
NURSING ACTIVITIES THAT CONTRIBUTE TO THE \\ QUALITY OF CARE: CONFIRMATORY FACTORIAL \\ ANALYSIS OF THE SCALE
}

\section{ACTIVIDADES DE ENFERMERÍA QUE CONTRIBUYEN PARA LA CALIDAD DE LOS CUIDADOS: ANÁLISIS FACTORIAL CONFIRMATORIA DE LA ESCALA}

\author{
Olga Maria Pimenta Lopes Ribeiro ${ }^{1}$ \\ Maria Manuela Ferreira Pereira da Silva Martins ${ }^{2}$ \\ Daisy Maria Rizatto Tronchin ${ }^{3}$ \\ Paulo João Figueiredo Cabral Teles ${ }^{4}$ \\ Letícia de Lima Trindade 5 \\ João Miguel Almeida Ventura da Silva ${ }^{6}$
}

Como citar este artigo: Ribeiro OMPL, Martins MMFPS, Tronchin DMR, Teles PJFC, Trindade LL, Silva JMAV. Atividades de enfermagem que contribuem para a qualidade dos cuidados: análise fatorial confirmatória da escala. Rev baiana de enferm. 2020:34:e36432.

Objetivo: analisar a estrutura fatorial da Escala de Percepção das Atividades de Enfermagem que Contribuem para a Qualidade dos Cuidados. Método: estudo metodológico com participação de 3.451 enfermeiros de 36 hospitais portugueses. Além da realização da análise fatorial confirmatória, para avaliação da confiabilidade do modelo fatorial obtido, utilizou-se o alfa de Cronbach e a confiabilidade compósita. Resultados: os pesos fatoriais da solução encontrada foram majoritariamente elevados; os valores dos índices de ajustamento do modelo foram razoáveis; o alfa de Cronbach foi elevado para a totalidade da escala e para cinco dimensões, sendo aceitável em apenas uma dimensão. A confiabilidade compósita também foi elevada em cinco dimensões, excepto em uma, considerada como aceitável. Todas as atividades apresentaram uma confiabilidade individual elevada. Conclusão: em comparação com a escala original, o modelo fatorial identificado contempla seis dimensões e não sete, produzindo uma escala confiável e válida, passível de aplicação no contexto hospitalar.

Descritores: Estudos de Validação. Análise Fatorial. Psicometria. Garantia da Qualidade dos Cuidados de Saúde. Cuidados de Enfermagem.

\footnotetext{
Enfermeira. Doutora em Ciências da Enfermagem. Professora Adjunta na Escola Superior de Enfermagem do Porto. Porto, Portugal. olgaribeiro@esenf.pt. https:// orcid.org/0000-000 I-9982-9537.

2 Enfermeira. Doutora em Ciências da Enfermagem. Professora Coordenadora na Escola Superior de Enfermagem do Porto. Porto, Portugal. https://orcid.org/00000003-1527-9940.

Enfermeira. Doutora em Ciências. Professora Associada da Escola de Enfermagem da Universidade de São Paulo. São Paulo, São Paulo, Brasil. https://orcid.org/00000003-3192-1956.

4 Doutor em Estatística. Professor Auxiliar na Faculdade de Economia da Universidade do Porto. Porto, Portugal. https://orcid.org/0000-000 I-7969- | 403.

Enfermeira. Doutora em Enfermagem. Docente na Universidade do Estado de Santa Catarina e no Programa de Pós-Graduação em Ciências da Saúde da Universidade Comunitária da Região de Chapecó. Chapecó, Santa Catarina, Brasil. https://orcid.org/0000-0002-7| I 9-0230.

6 Enfermeiro. Mestre em Ciências da Enfermagem. Enfermeiro no Centro Hospitalar Universitário São João. Porto, Portugal. https://orcid.org/0000-0002-8794-528X.
} 
Objective: to analyze the factorial structure of the Perception Scale of Nursing Activities that Contribute to the Quality of Care. Method: a methodological study with 3,451 nurses from 36 Portuguese hospitals. In addition to carrying out confirmatory factorial analysis, Cronbach's alpha and composite reliability were used to assess the reliability of the obtained factorial model. Results: the factorial weights of the solution found were mostly high; the values of the model's adjustment indexes were reasonable; Cronbach's alph a was elevated for the entire scale and five dimensions, being acceptable in only one dimension. The composite reliability was also high in five dimensions, except for one, considered acceptable. All activities showed high individual reliability. Conclusion: Compared to the original scale, the identified factorial model contemplates six dimensions and not seven, producing a reliable and valid scale, which can be applied in the hospital context.

Descriptors: Validation Study. Factor Analysis, Statistical. Psychometrics. Quality Assurance, Health Care. Nursing Care.

Objetivo: analizar la estructura factorial de la Escala de Percepción de las Actividades de Enfermería que Contribuyen para la Calidad de los Cuidados. Método: estudio metodológico con la participación de 3.451 enfermeros de 36 hospitales portugueses. Además de la realización del análisis factorial confirmatoria, para la evaluación de la fiabilidad del modelo factorial obtenido, se utilizó el alfa de Cronbach y la fiabilidad compuesta. Resultados: los pesos factoriales de la solución encontrada fueron mayoritariamente elevados; los valores de los indices de ajuste del modelo fueron razonables; el alfa de Cronbach fue elevado para la totalidad de la escala y para cinco dimensiones, siendo aceptable en apenas una dimensión. La fiabilidad compuesta también fue elevada en cinco dimensiones, excepto en una, considerada como aceptable. Todas las actividades presentaron una fiabilidad individual elevada. Conclusión: en comparación con la escala original, el modelo factorial identificado contiene seis dimensiones e no siete, produciendo una escala confiable y válida, pasible de aplicación en el contexto bospitalario.

Descriptores: Estudio de Validación. Análisis Factorial. Psicometría. Garantía de la Calidad de Atención de Salud. Atención de Enfermería.

\section{Introdução}

As questões da qualidade em saúde têm sido uma preocupação das organizações, bem como dos profissionais que nelas atuam. A par das significativas mudanças nas necessidades de saúde das populações, o elevado desenvolvimento tecnológico, a complexidade das dinâmicas de trabalho e as exigências cada vez maiores dos pacientes/usuários constituem, para os profissionais de saúde, importantes desafios ${ }^{(1-2)}$.

A evolução das ciências da saúde preconiza, além do desenvolvimento do conhecimento científico, a garantia da qualidade na prestação dos cuidados de saúde. Em resposta a esses dois pressupostos, em Portugal, tem-se defendido a importância dos padrões de qualidade e sua difusão no âmbito da prestação de cuidados, assim como o envolvimento dos profissionais de todo o Sistema Nacional de Saúde (SNS), de forma a potenciar a melhoria contínua da qualidade ${ }^{(3)}$. Ainda que o referido constitua um enorme desafio, é consensual que a qualidade da assistência deve ser priorizada pelas instituições de saúde e pelos profissionais que as integram ${ }^{(4)}$.
Em relação à Enfermagem, a defesa da qualidade dos cuidados prestados à população constituiu um dos propósitos da Ordem dos Enfermeiros desde a sua criação, no ano de $1998^{(5)}$. Nesse seguimento, já em 2001, no contexto português, foram publicados, pelo órgão regulador da profissão, Ordem dos Enfermeiros, os padrões de qualidade dos cuidados de enfermagem que simbolizam, para os enfermeiros, a referência para uma atuação profissional de excelência ${ }^{(6)}$.

$\mathrm{Na}$ área da saúde, é consensual que a busca pela melhoria contínua da qualidade está enraizada na atuação diária dos enfermeiros. No entanto, apesar de estarem claramente definidas as condições para se garantir a qualidade dos cuidados prestados, torna-se imperativo uma monitorização sistematizada, de maneira a fomentar e alcançar a cultura de qualidade ${ }^{(4,7)}$.

Nesse sentido, o emprego dos indicadores como medida de monitoramento e a avaliação da qualidade, incorporando a avaliação da qualidade da assistência de enfermagem por meio de instrumentos que analisem a percepção, as 
experiências e/ou satisfação dos clientes ${ }^{(4,8-9)}$, têm sido amplamente concretizadas. Todavia, ainda que a avaliação da qualidade do ponto de vista do paciente/usuário seja relevante ${ }^{(8)}$, conhecer a perspectiva dos enfermeiros sobre a concretização das atividades que contribuem para a qualidade dos cuidados prestados pode constituir uma oportunidade de promover o seu empoderamento em prol da melhoria contínua e da excelência do exercício profissional ${ }^{(10)}$.

Nos estudos desenvolvidos no contexto nacional e internacional, apesar da avaliação da percepção do profissional ser menos frequente, nota-se um interesse crescente na temática ${ }^{(11-13)}$. Além disso, uma vez que as particularidades do exercício profissional dos enfermeiros variam entre os diversos países, tem surgido a necessidade de construir e validar instrumentos ajustados à realidade dos distintos cenários ${ }^{(14)}$.

Em Portugal, no ano de 2014, alguns autores iniciaram o processo de construção de um instrumento cuja base conceptual foram os padrões de qualidade dos cuidados de enfermagem definidos pela Ordem dos Enfermeiros do país ${ }^{(6)}$. O referido instrumento, nominado como "Escala de Percepção das Atividades de Enfermagem que Contribuem para a Qualidade dos Cuidados (EPAECQC)" foi validado em 2014 em amostra de 775 enfermeiros de um hospital na região Norte de Portugal ${ }^{(15)}$. Decorrente do processo de validação, a Escala foi constituída por 25 itens distribuídos em sete dimensões.

Nesse estudo, a despeito do rigor metodológico na construção e validação da mencionada Escala, foi assumido pelos autores, como limitação, o fato de ter sido aplicada em um único hospital. Embora os resultados obtidos traduzissem que o instrumento apresentava propriedades psicométricas adequadas, com elevada consistência interna (alfa de Cronbach de $0,940)$ e com um valor de 0,942 no índice de Kaiser-Meyer-Olkin, os autores sugeriram a realização de estudos que fortalecessem as análises psicométricas e aprimorassem a estrutura fatorial da $\operatorname{Escala}^{(15)}$.

Assim, no âmbito de uma investigação nacional, conduzida de 2016 a 2018, estabeleceu-se como fundamental proceder à análise fatorial confirmatória e adensar as propriedades psicométricas da EPAECQC. Nesse seguimento, o objetivo deste estudo é analisar a estrutura fatorial da Escala de Percepção das Atividades de Enfermagem que Contribuem para a Qualidade dos Cuidados.

\section{Método}

Estudo metodológico com abordagem quantitativa, desenvolvido em 36 instituições hospitalares de Portugal continental. A população do estudo correspondeu aos enfermeiros que atuavam em serviços de cirurgia, medicina e medicina intensiva e urgência dos hospitais supracitados. Nesse contexto, após conhecidos os serviços onde a realização do estudo foi autorizada, identificou-se que a população elegível seria de 10.013 enfermeiros.

Para a obtenção da amostra, utilizou-se a técnica de amostragem não probabilística de conveniência. Como critérios de inclusão, foram definidos: exercer a atividade profissional no hospital, num espaço temporal igual ou superior a seis meses, em serviços de cirurgia, medicina ou medicina intensiva e urgência. Foram excluídos os enfermeiros que, no período da coleta de dados, estavam ausentes dos serviços por motivo de licença médica ou férias. Obteve-se uma amostra de 3.451 enfermeiros.

A coleta de dados realizou-se entre os meses de janeiro e março de 2016, mediante aplicação de um questionário constituído por duas partes: uma relativa à caracterização sociodemográfica e profissional dos enfermeiros e, outra, com a EPAECQC. Esta Escala é composta de 25 itens distribuídos por sete dimensões, a saber: organização dos cuidados de enfermagem (com dois itens de avaliação); satisfação do cliente, promoção da saúde e prevenção de complicações (com três itens cada dimensão); bem-estar e autocuidado e readaptação funcional (com quatro itens cada dimensão); e responsabilidade e rigor (com seis itens de avaliação). Os itens são respondidos por meio de uma escala do tipo Likert, com quatro graus, sendo atribuído 1 à opção 
"nunca", 2 à opção "poucas vezes", 3 à opção "às vezes" e 4 à opção "sempre”"(15).

Para a análise e o tratamento dos dados, usou-se o programa Statistical Package for the Social Sciences (SPSS), versão 22.0. Foram realizadas análises descritivas e psicométricas. Para avaliar a confiabilidade da escala, empregou-se o coeficiente alfa de Cronbach, a correlação item-total, a correlação inter-item e o coeficiente das duas metades de Guttman ${ }^{(16-17)}$. Posteriormente, procedeu-se ao cálculo da medida de adequação da amostragem de Kaiser-Meyer-Olkin (KMO), para cada atividade e para a escala total. Os valores esperados de KMO variam entre 0,5 e $1^{(16)}$.

Em seguida, no âmbito da análise fatorial confirmatória, para avaliar a qualidade do ajustamento do modelo proposto, foram utilizados os seguintes índices: Comparative Fit Index (CFI) com valor de aceitação igual ou superior a 0,9; Goodness of Fit Index (GFI) com valor de aceitação igual ou superior a 0,85; Adjusted Goodness of Fit Index (AGFI) com um valor de aceitação igual ou superior a 0,80; Root Mean Square Residual (RMR) com valor de aceitação menor ou igual a 0,1; Root Mean Square Error of Approximation (RMSEA) com valor de aceitação menor que 0,1; e, também, Modified Expected Cross-Validation Index (MECVI) ${ }^{(16-17)}$.

Em relação à avaliação da confiabilidade do modelo fatorial obtido, utilizou-se o coeficiente alfa de Cronbach e a confiabilidade compósita. Relativo ao alfa de Cronbach, considerou-se que um valor superior a 0,8 significaria que a consistência interna seria boa, sendo o valor ideal situado entre 0,8 e $0,9^{(16,18)}$. Por outro lado, no que concerne à confiabilidade compósita, julgou-se que um valor igual ou superior a 0,7 seria indicador de uma confiabilidade de construto apropriada, embora valores inferiores ainda pudessem ser aceitáveis ${ }^{(17)}$.

Com o intuito de avaliar a validade fatorial, calcularam-se os pesos da regressão estandardizados de cada atividade, bem como o quadrado desses pesos, designados por confiabilidade individual. Considerou-se que a confiabilidade individual é apropriada para um valor igual ou superior a $0,25^{(17)}$. Por fim, a validade convergente de cada dimensão foi medida por meio da sua Variância Extraída Média (VEM), sendo considerada adequada quando igual ou superior a $0,5^{(17)}$.

Uma vez que o presente estudo está integrado a uma investigação nacional, foi inicialmente aprovado pelo Comité de Ética para a Saúde de uma instituição hospitalar do Norte de Portugal, cujo número do Parecer foi 98-15. Posteriormente, teve a aprovação dos Comités de Ética e dos Conselhos de Administração das 36 instituições hospitalares envolvidas. Importa referir que, após terem sido informados sobre o estudo, os participantes assinaram o consentimento informado. Foi garantido o anonimato e a confidencialidade das informações coletadas.

\section{Resultados}

Dos 3.451 participantes, 2.659 (77,1\%) eram do gênero feminino, com uma idade mínima de 22 anos e máxima de 62 anos. A média de idade correspondeu a 36,4 anos, com desvio padrão de 8,3. Relativamente ao estado civil, 2.109 enfermeiros $(61,1 \%)$ eram casados ou viviam em união estável e 1.168 (33,8\%) eram solteiros. Com relação ao exercício profissional, 2.633 (76,3\%) eram enfermeiros de cuidados gerais (cujo nível de formação é a graduação), 686 (19,9\%) enfermeiros especialistas (que, além da graduação, possuíam formação numa das áreas de especialização reconhecidas pela Ordem dos Enfermeiros de Portugal) e 132 (3,8\%) enfermeiros gestores (possuíam graduação, especialização e formação na área da gestão). No que se refere à unidade de atuação, 1.526 (44,2\%) trabalhavam em serviços de medicina, 1.161 (33,7\%) em serviços de cirurgia e 764 (22,1\%) em serviços de medicina intensiva e urgência.

Quanto à distribuição dos enfermeiros segundo as Regiões de Saúde a que pertenciam os hospitais onde exerciam funções, 1.492 (43,2\%) trabalhavam no Norte, 829 (24,0\%) em Lisboa e Vale do Tejo, 771 (22,3\%) no Centro, 213 (6,2\%) no Alentejo e 146 (4,2\%) no Algarve.

No que se refere aos resultados decorrentes da aplicação da EPAECQC, primeiramente, foi verificado se os dados eram apropriados para 
fazer a análise fatorial. As correlações item-total corrigidas variaram entre 0,827 e 0,999 , com uma correlação média de 0,955 , valores muito elevados e que mostraram uma forte homogeneidade dos itens (atividades) constituintes da Escala (Tabela 1).

Tabela 1 - Correlações item-total corrigidas. Porto, Portugal - 2016. ( N=3.451)

\begin{tabular}{l|c||c|c||c|c}
\hline Atividade & Correlação & Atividade & Correlação & Atividade & Correlação \\
\hline 1 & 0,968 & 10 & 0,997 & 19 & 0,928 \\
2 & 0,951 & 11 & 0,981 & 20 & 0,827 \\
3 & 0,936 & 12 & 0,982 & 21 & 0,890 \\
4 & 0,874 & 13 & 0,987 & 22 & 0,900 \\
5 & 0,914 & 14 & 0,990 & 23 & 0,959 \\
6 & 0,909 & 15 & 0,976 & 24 & 0,998 \\
7 & 0,994 & 16 & 0,972 & 25 & 0,997 \\
8 & 0,993 & 17 & 0,967 & & \\
9 & 0,998 & 18 & 0,999 & & \\
\hline
\end{tabular}

Fonte: Elaboração própria.

Em seguida, ao analisar a confiabilidade da Escala, foram utilizados o coeficiente alfa de Cronbach, a correlação item-total, a correlação inter-item e o coeficiente das duas metades de Guttman. Com relação ao alfa de Cronbach, dividindo a escala em duas partes com um número de atividades o mais próximo possível, os valores obtidos foram considerados elevados. Na primeira parte da escala, com um total de 13 itens, o valor de alfa de Cronbach correspondeu a 0,994; na segunda parte, com 12 itens, o valor de alfa de Cronbach foi de 0,990.

A correlação item-total corrigida média foi de 0,955 , valor apontado como elevado. A correlação inter-item média foi de 0,304, classificada como muito aceitável, uma vez que havia um número expressivo de correlações moderadas ou elevadas, que evidenciaram validade convergente. Por fim, o coeficiente das duas metades de Guttman foi de 0,988, também um valor muito elevado. Em resumo, todos esses coeficientes levaram a concluir que a consistência da Escala era muito boa.

A medida de adequação da amostragem de Kaiser-Meyer-Olkin, para cada atividade e para a totalidade da Escala (valor global), encontra-se na Tabela 2. O valor global foi de 0,902, correspondendo ao grau de muito elevado. Os valores para cada atividade são todos elevados ou muito elevados, mostrando-se bem superiores a 0,5. Importa referir que, em 16 atividades, o valor foi superior a 0,9 , sendo em todas as outras atividades superior a 0,7 . Em resumo, pode-se afirmar que a fatorabilidade da matriz de correlações foi muito boa, sendo apropriado efetuar uma análise fatorial com esses dados.

Tabela 2 - Medida de adequação da amostragem Kaiser-Meyer-Olkin. Porto, Portugal - 2016. (N=3.451)

\begin{tabular}{l|c||c|c||c|c}
\hline Atividade & $\begin{array}{c}\text { Kaiser-Meyer- } \\
\text { Olkin }\end{array}$ & Atividade & $\begin{array}{c}\text { Kaiser-Meyer- } \\
\text { Olkin }\end{array}$ & Atividade & $\begin{array}{c}\text { Kaiser-Meyer- } \\
\text { Olkin }\end{array}$ \\
\hline 1 & 0,960 & 10 & 0,944 & 19 & 0,921 \\
2 & 0,952 & 11 & 0,909 & 20 & 0,721 \\
3 & 0,953 & 12 & 0,904 & 21 & 0,840 \\
4 & 0,948 & 13 & 0,942 & 22 & 0,723 \\
5 & 0,934 & 14 & 0,970 & 23 & 0,855 \\
6 & 0,933 & 15 & 0,920 & 24 & 0,910 \\
7 & 0,872 & 16 & 0,931 & 25 & 0,934 \\
8 & 0,814 & 17 & 0,938 & & 0,902 \\
9
\end{tabular}

Fonte: Elaboração própria. 
Assim, realizou-se uma análise fatorial com extração de fatores pelo método de componentes principais, tendo sido necessário determinar, em primeiro lugar, o número de fatores a reter. As regras habitualmente utilizadas para selecionar o número de fatores a reter na análise conduzem a diferentes soluções. Com efeito, uma dessas regras consiste em selecionar os fatores cujos valores próprios associados sejam superiores a um (regra de Kaiser), sendo o sexto fator o último que a cumpre, pelo que esta regra apontaria para uma solução com seis fatores, um número satisfatório, explicando $65,29 \%$ da variância total, o que é também muito aceitável. Uma segunda regra consiste em reconstituir $80 \%$ da variância total (regra de Pearson), o que conduz a uma solução com 12 fatores. O conjunto dos primeiros 12 fatores explica 80,03\% da variância total, o que é altamente elevado e, por isso, não é adequado. Finalmente, a terceira regra empregada é baseada no scree plot, no qual se retém o número de fatores em que ocorre a maior quebra da percentagem da variância explicada (regra de Cattell), o que conduz a reter quatro fatores, que explicam apenas 56,7\% da variância total.

Ponderadas as três possibilidades, adotou-se a solução com seis fatores, pois explica uma percentagem aceitável da variância total $(65,29 \%)$ e é a melhor solução no tocante à interpretação e significado dos fatores. Além disso, a consideração de um número de fatores mais elevado conduziu a fatores desnecessários ou a um acréscimo quase nulo da variância explicada. A consideração de um número inferior não é suficiente, pois faz com que a representação de várias atividades seja de má qualidade e diminua a percentagem da variância explicada.

Os resultados da análise fatorial forçada a seis fatores, seguida de rotação varimax e normalização de Kaiser, estão apresentados na Tabela 3, na qual se indicam os pesos fatoriais das diferentes atividades em cada fator, encontrando-se em negrito o peso mais elevado de cada atividade. Para mais fácil leitura e interpretação dos resultados, as atividades estão indicadas pela ordem do fator em que saturam e não pela ordem da Escala original. Refira-se que os pesos fatoriais da solução obtida apresentam geralmente valores elevados, muito elevados ou, pelo menos, aceitáveis (alguns casos apenas), o que permite concluir novamente que a solução fatorial obtida tem boa qualidade. Além disso, quanto às comunalidades, isto é, a percentagem da variância de cada atividade explicada conjuntamente pelos seis fatores extraídos, verifica-se que é majoritariamente superior a 50\% em todas as atividades, com apenas cinco exceções (e quatro destas estão extremamente próximas de 50\%), sendo boa em algumas atividades e elevada em outras, o que significa, mais uma vez, que os resultados desta análise fatorial são de boa qualidade.

Tabela 3 - Escala de Percepção das Atividades de Enfermagem que Contribuem para a Qualidade dos Cuidados - estrutura fatorial. Porto, Portugal - 2016. (N=3.451)

(continua)

\begin{tabular}{l|c|c|c|c|c|c|c}
\hline & Fator & Fator & Fator & Fator & Fator & Fator & Comunalidades \\
\hline Satisfação do cliente - Item 1 & $\mathbf{0 , 6 4 0}$ & 0,244 & 0,116 & 0,080 & 0,063 & $-0,014$ & 0,494 \\
Satisfação do cliente - Item 2 & $\mathbf{0 , 6 5 1}$ & 0,168 & 0,142 & 0,019 & 0,038 & 0,109 & 0,486 \\
Satisfação do cliente - Item 3 & $\mathbf{0 , 7 2 9}$ & 0,066 & 0,169 & 0,190 & $-0,029$ & 0,113 & 0,614 \\
Promoção da saúde - Item 1 & $\mathbf{0 , 4 6 2}$ & $-0,003$ & 0,043 & 0,323 & 0,347 & 0,104 & 0,451 \\
Promoção da saúde - Item 2 & $\mathbf{0 , 6 3 6}$ & 0,015 & 0,262 & 0,312 & 0,077 & 0,103 & 0,587 \\
Promoção da saúde - Item 3 & $\mathbf{0 , 6 4 6}$ & 0,031 & 0,269 & 0,308 & 0,075 & 0,170 & 0,620 \\
Responsabilidade e rigor - Item 1 & $-0,001$ & $\mathbf{0 , 7 9 1}$ & $-0,028$ & 0,036 & 0,227 & $-0,016$ & 0,691 \\
Responsabilidade e rigor - Item 2 & 0,335 & $\mathbf{0 , 6 8 5}$ & 0,225 & 0,139 & $-0,067$ & 0,214 & 0,714 \\
Responsabilidade e rigor - Item 3 & $-0,035$ & $\mathbf{0 , 7 7 5}$ & $-0,012$ & 0,020 & 0,284 & 0,031 & 0,686 \\
Responsabilidade e rigor - Item 4 & 0,259 & $\mathbf{0 , 7 4 2}$ & 0,196 & 0,133 & 0,026 & 0,229 & 0,680 \\
Responsabilidade e rigor - Item 5 & 0,162 & $\mathbf{0 , 5 1 1}$ & 0,224 & 0,219 & 0,019 & 0,085 & 0,760 \\
Responsabilidade e rigor - Item 6 & 0,387 & $\mathbf{0 , 4 1 7}$ & 0,287 & 0,210 & $-0,124$ & 0,350 & 0,840 \\
Bem-estar e autocuidado - Item 1 & 0,217 & 0,142 & $\mathbf{0 , 6 9 0}$ & 0,171 & 0,319 & 0,079 & 0,694
\end{tabular}


Tabela 3 - Escala de Percepção das Atividades de Enfermagem que Contribuem para a Qualidade dos Cuidados - estrutura fatorial. Porto, Portugal - 2016. (N=3.451)

(conclusão)

\begin{tabular}{|c|c|c|c|c|c|c|c|}
\hline Atividades & $\begin{array}{c}\text { Fator } \\
1 \\
\end{array}$ & $\begin{array}{c}\text { Fator } \\
2 \\
\end{array}$ & $\begin{array}{c}\text { Fator } \\
3 \\
\end{array}$ & $\begin{array}{c}\text { Fator } \\
4\end{array}$ & $\begin{array}{c}\text { Fator } \\
5\end{array}$ & $\begin{array}{c}\text { Fator } \\
6\end{array}$ & Comunalidades \\
\hline Bem-estar e autocuidado - Item 2 & 0,153 & 0,094 & 0,770 & 0,178 & 0,315 & 0,062 & 0,480 \\
\hline Bem-estar e autocuidado - Item 3 & 0,325 & 0,125 & 0,799 & 0,240 & 0,096 & 0,115 & 0,753 \\
\hline Bem-estar e autocuidado - Item 4 & 0,282 & 0,154 & 0,714 & 0,260 & 0,028 & 0,112 & 0,773 \\
\hline Readaptação funcional - Item 1 & 0,114 & 0,206 & 0,261 & 0,572 & 0,164 & 0,045 & 0,745 \\
\hline Readaptação funcional - Item 2 & 0,130 & 0,090 & 0,080 & 0,831 & 0,172 & 0,036 & 0,769 \\
\hline Readaptação funcional - Item 3 & 0,336 & 0,118 & 0,283 & 0,734 & 0,017 & 0,163 & 0,710 \\
\hline Readaptação funcional - Item 4 & 0,344 & 0,123 & 0,287 & 0,710 & 0,020 & 0,156 & 0,680 \\
\hline $\begin{array}{l}\text { Prevenção de complicações - } \\
\text { Item } 1\end{array}$ & 0,067 & 0,121 & 0,088 & 0,086 & 0,808 & 0,065 & 0,702 \\
\hline $\begin{array}{l}\text { Prevenção de complicações - } \\
\text { Item } 2\end{array}$ & 0,030 & 0,117 & 0,150 & 0,087 & $\mathbf{0 , 8 1 7}$ & 0,043 & 0,685 \\
\hline $\begin{array}{l}\text { Prevenção de complicações - } \\
\text { Item } 3\end{array}$ & 0,048 & 0,130 & 0,204 & 0,091 & 0,785 & $-0,003$ & 0,728 \\
\hline $\begin{array}{l}\text { Organização cuidados } \\
\text { enfermagem - Item } 1\end{array}$ & 0,075 & 0,138 & 0,045 & 0,083 & 0,150 & 0,844 & 0,393 \\
\hline $\begin{array}{l}\text { Organização cuidados } \\
\text { enfermagem - Item } 2\end{array}$ & 0,258 & 0,161 & 0,177 & 0,149 & $-0,016$ & 0,751 & 0,588 \\
\hline
\end{tabular}

Fonte: Elaboração própria.

No âmbito da avaliação da qualidade do ajustamento do modelo proposto, o Comparative Fit Index (CFI) foi de 0,845, indicando um ajustamento que pode ser considerado quase aceitável. De forma semelhante, o Goodness of Fit Index (GFI) e o Adjusted Goodness of Fit Index (AGFI) foram de 0,842 e 0,803, respetivamente, valores aceitáveis, embora não cheguem ainda a ser bons. O Root Mean Square Residual (RMR) foi de 0,021, um valor estimado como baixo, tendo em consideração a escala de resposta das atividades, o que é favorável. O Root Mean Square Error of Approximation (RMSEA) foi de 0,087, um valor também razoável, considerando que o ajustamento é aceitável para um valor inferior a 0,1 e bom para um valor inferior a 0,06. Por fim, para comparação com outros modelos, confirmou-se também o Modified Expected Cross-Validation Index (MECVI), que assumiu o valor 2,083. Em síntese, a qualidade do ajustamento é razoável, próxima de ser considerada boa.
Posteriormente, procedeu-se à avaliação da confiabilidade da nova estrutura da Escala, utilizando, para tal, a consistência interna, o coeficiente alfa de Cronbach e a confiabilidade compósita para a totalidade da Escala e para as seis dimensões agora identificadas.

O valor do alfa de Cronbach para a totalidade da Escala foi de 0,917, o que é muito elevado e mostra uma consistência interna da Escala muito forte. Evidenciou-se também que a consistência de todas as dimensões foi alta, uma vez que os respetivos valores de alfa de Cronbach foram superiores a 0,8, com exceção da sexta dimensão, em que o valor foi aceitável. Com relação à confiabilidade compósita, constatou-se que foi elevada para todas as dimensões, exceto para a sexta, em que foi ainda aceitável (Tabela 4). Em conclusão, quer a escala global, quer as dimensões identificadas revelam uma boa confiabilidade e consistência interna. 
Tabela 4 - Escala de Percepção das Atividades de Enfermagem que Contribuem para a Qualidade dos Cuidados - confiabilidade da nova estrutura das dimensões. Porto, Portugal - 2016. (N=3.451)

\begin{tabular}{lcc}
\hline Dimensões & alfa Cronbach & Confiabilidade Compósita \\
\hline 1 - Satisfação do cliente e promoção da saúde & 0,801 & 0,803 \\
2 - Responsabilidade e rigor & 0,809 & 0,830 \\
3 - Bem-estar e autocuidado & 0,876 & 0,880 \\
4 - Readaptação funcional & 0,837 & 0,841 \\
5 - Prevenção de complicações & 0,820 & 0,822 \\
6 - Organização dos cuidados de enfermagem & 0,650 & 0,672 \\
\hline
\end{tabular}

Fonte: Elaboração própria.

Para avaliar a validade fatorial, a Tabela 5 mostra os pesos da regressão estandardizados de cada atividade nas diversas dimensões e o quadrado desses pesos, designados por confiabilidade individual. Todas as atividades apresentaram confiabilidade individual superior a 0,25 e, portanto, apropriada, sendo muito superior na maioria delas, achados que permitem afirmar que todas as dimensões apresentam validade fatorial.

Tabela 5 - Escala de Percepção das Atividades de Enfermagem que Contribuem para a Qualidade dos Cuidados - pesos da regressão estandardizados nas dimensões. Porto, Portugal - 2016. $(\mathrm{N}=3.451)$

\begin{tabular}{|c|c|c|}
\hline Dimensões e atividades & $\begin{array}{c}\text { Peso da } \\
\text { regressão }\end{array}$ & $\begin{array}{c}\text { Confiabilidade } \\
\text { individual }\end{array}$ \\
\hline \multicolumn{3}{|c|}{ Dimensão 1: Satisfação do cliente e promoção da saúde } \\
\hline Satisfação do cliente - Item 1 & 0,521 & 0,271 \\
\hline Satisfação do cliente - Item 2 & 0,520 & 0,270 \\
\hline Satisfação do cliente - Item 3 & 0,659 & 0,434 \\
\hline Promoção da saúde - Item 1 & 0,531 & 0,282 \\
\hline Promoção da saúde - Item 2 & 0,765 & 0,585 \\
\hline Promoção da saúde - Item 3 & 0,795 & 0,632 \\
\hline \multicolumn{3}{|l|}{ Dimensão 2: Responsabilidade e rigor } \\
\hline Responsabilidade e rigor - Item 1 & 0,562 & 0,316 \\
\hline Responsabilidade e rigor - Item 2 & 0,800 & 0,640 \\
\hline Responsabilidade e rigor - Item 3 & 0,578 & 0,334 \\
\hline Responsabilidade e rigor - Item 4 & 0,848 & 0,719 \\
\hline Responsabilidade e rigor - Item 5 & 0,533 & 0,284 \\
\hline Responsabilidade e rigor - Item 6 & 0,670 & 0,449 \\
\hline \multicolumn{3}{|l|}{ Dimensão 3: Bem-estar e autocuidado } \\
\hline Bem-estar e autocuidado - Item 1 & 0,749 & 0,561 \\
\hline Bem-estar e autocuidado - Item 2 & 0,793 & 0,629 \\
\hline Bem-estar e autocuidado - Item 3 & 0,900 & 0,810 \\
\hline Bem-estar e autocuidado - Item 4 & 0,771 & 0,594 \\
\hline \multicolumn{3}{|l|}{ Dimensão 4: Readaptação funcional } \\
\hline Readaptação funcional - Item 1 & 0,563 & 0,317 \\
\hline Readaptação funcional - Item 2 & 0,685 & 0,469 \\
\hline Readaptação funcional - Item 3 & 0,880 & 0,774 \\
\hline Readaptação funcional - Item 4 & 0,862 & 0,743 \\
\hline \multicolumn{3}{|l|}{ Dimensão 5: Prevenção de complicações } \\
\hline Prevenção de complicações - Item 1 & 0,700 & 0,490 \\
\hline Prevenção de complicações - Item 2 & 0,828 & 0,686 \\
\hline Prevenção de complicações - Item 3 & 0,805 & 0,648 \\
\hline \multicolumn{3}{|c|}{ Dimensão 6: Organização dos cuidados de enfermagem } \\
\hline Organização dos cuidados enfermagem - Item 1 & 0,589 & 0,347 \\
\hline Organização dos cuidados enfermagem - Item 2 & 0,824 & 0,679 \\
\hline
\end{tabular}

Fonte: Elaboração própria. 
A validade convergente de cada dimensão foi medida por meio da sua Variância Extraída Média (VEM). Com relação à dimensão satisfação do cliente e promoção da saúde, a VEM foi de 0,413; na responsabilidade e rigor, foi de 0,457; no bem-estar e autocuidado, foi de 0,649; na readaptação funcional, foi de 0,576; na prevenção de complicações, foi de 0,609; e na organização dos cuidados de enfermagem, foi de 0,531 . Verificou-se, assim, que as quatro últimas dimensões têm uma VEM claramente superior a 0,5 , indicador de uma validade convergente adequada. Pelo contrário, as duas primeiras têm uma VEM inferior a 0,5 , mas muito próxima deste valor, o que indica uma validade convergente ainda aceitável.

\section{Discussão}

A EPAECQC foi construída para atender a uma fragilidade relacionada com a inexistência de instrumentos que permitissem avaliar a percepção dos enfermeiros com relação à qualidade da assistência de enfermagem, tendo por referência os padrões de qualidade legalmente definidos em Portugal ${ }^{(15)}$. Decorrente da especificidade da Escala, além de ser usada no contexto nacional ${ }^{(12,19)}$, tem despertado a atenção de outros investigadores, interessados na sua validação em outros contextos ${ }^{(11)}$.

Embora o instrumento tenha como referencial teórico os padrões de qualidade dos cuidados de enfermagem ${ }^{(6)}$, tornou-se premente a realização de estudos para validar a sua estrutura fatorial, bem como a sua consistência interna. O fato de este estudo ter sido realizado em 36 instituições hospitalares distribuídas pelas 5 regiões de Portugal continental, constituiu uma excelente oportunidade de fortalecer as análises psicométricas e melhorar a estrutura da Escala. Nesse sentido, além de terem sido analisadas as correlações, a consistência interna e a qualidade do ajustamento obtido, procurou-se comparar estes resultados com os do estudo relativo à validação da Escala original ${ }^{(15)}$. Isso é importante também para identificação de necessidade de ajustes dos instrumentos dessa natureza, conforme as mudanças que podem emergir na práxis de Enfermagem.

Em relação à análise da estrutura fatorial da escala, evidenciou-se que o primeiro fator apresentou pesos fatoriais elevados das atividades incluídas nas dimensões satisfação do cliente e promoção da saúde, pelo que se optou por renomear este fator como dimensão da satisfação do cliente e promoção da saúde. Embora a satisfação do cliente e a promoção da saúde constituam enunciados descritivos distintos ${ }^{(6)}$, é interessante que estes dois tipos de atividades saturem no mesmo fator, pois isso significa que elas estão associadas. Assim, conclui-se que as atividades dos enfermeiros que conduzem à promoção da saúde estão estreitamente ligadas às que proporcionam satisfação ao cliente.

Esses achados indiciam aspectos importantes no contexto das práticas dos enfermeiros, uma vez que subsidiam atividades a serem valorizadas na assistência e na gestão em saúde e enfermagem. O referido ganha ainda mais relevância quando outros estudos ponderam sobre a importância de se mensurar diretrizes para os serviços com base nos motivos de satisfação dos clientes e de suas necessidades ${ }^{(20-21)}$.

Nesse contexto, o primeiro fator passa a integrar as atividades que se referem ao respeito pelos desejos, valores, crenças e capacidades dos clientes, à empatia nas interações que se estabelecem com os clientes e ao envolvimento dos conviventes significativos no processo de cuidados (dimensão "satisfação do cliente" na escala original). Inclui ainda as atividades relacionadas com a identificação de situações de saúde e os recursos dos pacientes/usuários, a otimização do internamento, para promover estilos de vida saudáveis e a disponibilização de informação geradora de aprendizagem cognitiva e de novas capacidades pelos clientes (dimensão "promoção da saúde" na escala original) ${ }^{(15)}$.

$\mathrm{Na}$ sequência de investigações anteriores, embora seja conhecido que as atividades integradas na dimensão promoção da saúde são mais frequentemente concretizadas pelos enfermeiros da Atenção Primária à Saúde do que pelos enfermeiros do contexto hospitalar ${ }^{(22)}$, os achados 
desta análise fatorial exigem desses profissionais uma reflexão que poderá culminar numa mudança de paradigma.

Efetivamente, se a satisfação do cliente pode estar relacionada com as atividades integradas na dimensão promoção da saúde, será lógico continuar, no contexto hospitalar, a não priorizar essas intervenções? Além disso, o fato de constituírem essencialmente intervenções do domínio autônomo da profissão, torna ainda mais evidente a necessidade de os enfermeiros atuarem para capacitar os clientes, de modo a torná-los agentes ativos no seu processo de saúde/doença, proporcionando-lhes a ajuda profissional de que necessitam e, simultaneamente, responsabilizando-os pelo seu projeto de saúde e pela adoção de estilos de vida saudáveis ${ }^{(22)}$. Consequentemente, é possível que esta mudança de paradigma tenha repercussão não só ao nível da satisfação do cliente, mas também da própria visibilidade da profissão, o que sinaliza a posição social que ocupa ${ }^{(20)}$.

O segundo fator apresentou pesos fatoriais elevados das atividades incluídas na responsabilidade e rigor, mantendo, por isso, a designação da respetiva dimensão no instrumento original. De forma semelhante, as restantes dimensões (fatores) apresentaram pesos fatoriais elevados apenas em relação às atividades nelas integradas, continuando, assim, com a designação da Escala original: bem-estar e autocuidado, readaptação funcional, prevenção de complicações e organização dos cuidados de enfermagem. Esta correspondência perfeita entre os fatores e os tipos de atividades, comprova que a Escala encontra-se muito bem construída, não existindo sobreposições nem repetições das atividades em diferentes fatores.

Com relação à consistência interna, na versão original da Escala, o alfa de Cronbach foi de 0,940 para a escala total. Nas sete dimensões, atingiu valores razoáveis ou bons: na dimensão satisfação do cliente, foi de 0,744 ; na promoção da saúde, foi de 0,740; na prevenção de complicações, foi de 0,779; no bem-estar e autocuidado, foi de 0,862; na readaptação funcional, foi de 0,830; na organização dos cuidados de enfermagem, foi de 0,684 ; e na responsabilidade e rigor, foi de $0,855^{(15)}$. Com a nova estrutura fatorial, apesar de o valor de alfa de Cronbach para a escala total ser ligeiramente inferior $(0,917) \mathrm{em}$ quatro dimensões, o valor foi superior: na satisfação do cliente e promoção da saúde $(0,801)$; no bem-estar e autocuidado $(0,876)$; na readaptação funcional $(0,837)$; e na prevenção de complicações $(0,820)$. Embora não exista consenso entre os diversos autores, os valores de alfa de Cronbach mais recomendados situam-se entre 0,80 e $0,90^{(18,23)}$, o que está em consonância com os valores obtidos em cinco das dimensões da nova estrutura fatorial.

$\mathrm{Na}$ dimensão organização dos cuidados de enfermagem, o alfa de Cronbach obteve o menor valor $(0,650)$, o que também se verificou na versão original da Escala $(0,684)^{(15)}$. Num estudo realizado na Turquia, com o objetivo de validar a Escala de Percepção das Atividades de Enfermagem que Contribuem para a Qualidade dos Cuidados ${ }^{(11)}$, o alfa de Cronbach relativo à dimensão organização dos cuidados de enfermagem também foi o menor comparativamente com todas as outras dimensões $(0,716)$. Tal constatação pode dever-se ao fato de esta dimensão incluir apenas duas atividades, até porque é conhecido que, quando uma dimensão inclui poucos itens, o valor de alfa de Cronbach é frequentemente baixo, o que não significa necessariamente baixa consistência ${ }^{(18,23)}$. Ainda assim, é de destacar que tanto na versão original como na versão atual da Escala, o valor de alfa de Cronbach é aceitável ${ }^{(23)}$.

Atendendo a que a confiabilidade compósita igual ou superior a 0,7 é indicadora de uma confiabilidade de constructo apropriada ${ }^{(17)}$, constatou-se que a confiabilidade compósita do modelo obtido é muita alta para todas as dimensões, exceto na organização dos cuidados de enfermagem, onde foi aceitável.

Quanto à análise confirmatória da nova estrutura da Escala, além de os pesos fatoriais apresentarem valores elevados, muito elevados ou, pelo menos, aceitáveis, a qualidade do ajustamento foi razoável, próxima de ser considerada boa. Atendendo a que o ajustamento é aceitável para um CFI maior ou igual a 0,9 , é importante destacar que o coeficiente obtido encontra-se muito 
próximo desse valor $(0,845)^{(16)}$. Com relação ao GFI e ao AGFI, considerando que valores superiores a 0,85 e 0,8 , respetivamente, indicam um bom ajustamento, é relevante salientar que o coeficiente obtido no GFI foi de 0,842, próximo de 0,85 , sendo o AGFI de 0,803 já superior ao valor de referência. Os valores obtidos por meio de outros testes de adequação também foram favoráveis $(\mathrm{RMR}=0,021 \text {; RMSEA }=0,087)^{(16-17)}$.

Por fim, considerando que a confiabilidade individual é apropriada para um valor igual ou superior a 0,25 , constatou-se que todas as atividades apresentaram uma confiabilidade superior a esse valor, sendo muito superior na maioria delas. Logo, pode-se afirmar que as seis dimensões propostas apresentaram validade fatorial ${ }^{(17)}$.

$\mathrm{Na}$ sequência do mencionado, o novo modelo fatorial identificado mostrou uma boa qualidade, confiabilidade e validade, pelo que se conclui que é apropriado, evidenciando potencial de aceitação. Não obstante o contributo deste estudo para melhorar a performance da EPAECQC, assume-se como limitação a técnica de amostragem ter sido não probabilística de conveniência. De fato, embora façam parte da amostra, enfermeiros de diversos contextos, o perfil dos que aceitaram e decidiram participar pode ter determinado a nova estrutura fatorial, sugerindo-se, assim, o desenvolvimento de estudos que usem a versão da Escala que decorre dessa análise fatorial.

Acresce ainda que o ajuste indicado na composição da Escala, além de constituir um estímulo para os profissionais de enfermagem atuarem em consonância com todos os enunciados descritivos, vem reforçar, mais uma vez, a necessidade das práticas de promoção da saúde serem devidamente incorporadas no exercício profissional dos enfermeiros no contexto hospitalar $^{(19)}$, até porque poderão ser determinantes para a satisfação do cliente, bem como para promover a visibilidade da profissão.

\section{Conclusão}

$\mathrm{Na}$ sequência da análise fatorial realizada e das medidas de ajuste implementadas, emerge uma alteração no número de dimensões da
EPAECQC. Enquanto a Escala original contempla sete dimensões, a proposta que sobressaiu deste estudo tem seis dimensões, sugerindo a possibilidade de agrupar num único fator as dimensões "satisfação do cliente" e "promoção da saúde". Nas demais dimensões, a correspondência ajustada entre elas e as respectivas atividades evidencia que o instrumento está adequadamente construído. Na prática, o fato de as atividades inerentes à "satisfação do cliente" e à "promoção da saúde" integrarem a mesma dimensão exige uma mudança de paradigma no contexto hospitalar, de modo a que fique fundamentalmente centrado no domínio autônomo da profissão.

Os resultados da análise fatorial confirmatória justificam a aceitação do novo modelo, sendo importante que se pondere o reajuste sugerido quanto ao número de dimensões. Por conseguinte, sugere-se a realização de investigações empregando a Escala, com o modelo fatorial proposto, o que permitirá comparar os achados aos estudos que foram conduzidos com a utilização da versão original.

\section{Colaborações:}

1 - concepção, projeto, análise e interpretação dos dados: Olga Maria Pimenta Lopes Ribeiro, Maria Manuela Ferreira Pereira da Silva Martins, Daisy Maria Rizatto Tronchin e Paulo João Figueiredo Cabral Teles;

2 - redação do artigo e revisão crítica relevante do conteúdo intelectual: Olga Maria Pimenta Lopes Ribeiro, Letícia de Lima Trindade e João Miguel Almeida Ventura da Silva;

3 - aprovação final da versão a ser publicada: Olga Maria Pimenta Lopes Ribeiro, Maria Manuela Ferreira Pereira da Silva Martins, Daisy Maria Rizatto Tronchin, Paulo João Figueiredo Cabral Teles, Letícia de Lima Trindade e João Miguel Almeida Ventura da Silva.

\section{Referências}

1. Feiten AM, Coelho TR. Quality management in service organizations: barriers and success factors. Rev Adm FACES Journal. 2019;18(3):56-71. DOI: https://doi.org/10.21714/ 1984-6975FACES2019V18N3ART6619 
2. Gaalan K, Kunaviktikul W, Akkadechanunt T, Wichaikhum OA, Turale S. Factors predicting quality of nursing care among nurses in tertiary care hospitals in Mongolia. Int Nurs Rev. 2019;66(2):176-82. DOI: https://doi.org/10.1111/ inr. 12502

3. Fronteira I, Jesus EH, Dussault G. Nursing in Portugal in the National Health Service at 40. Ciênc saúde coletiva. 2020;25(1):273-82. DOI: http:// dx.doi.org/10.1590/1413-81232020251.28482019

4. Caldana G, Gabriel CS, Rocha FLR, Bernardes A, Françolin L, Costa DB. Evaluating the quality of nursing care at a private hospital. Rev Eletr Enf. 2013;15(4):915-22. DOI: https://doi.org/10.5216/ ree.v15i4.19655

5. Cantante AP, Fernandes HI, Teixeira MJ, Frota MA, Rolim KM, Albuquerque FH. Health Systems and Nursing Skills in Portugal. Ciênc saúde coletiva. 2019;25(1):261-72. DOI: https://doi. org/10.1590/1413-81232020251.27682019

6. Ordem dos Enfermeiros. Padrões de qualidade dos cuidados de enfermagem, enquadramento conceptual.Enunciadosdescritivos[Internet].Lisboa: Ordem dos Enfermeiros; 2012 [cited 2020 Mar 13]. Available from: https://www.ordemenfermeiros. pt/media/8903/divulgar-padroes-de-qualidadedos-cuidados.pdf

7. Organisation for Economic Co-operation and Development. Caring for quality in health: Lessons learnt from 15 reviews of health care quality [Internet]. Paris: OECD; 2017 [cited 2020 Mar 8]. Available from: http://www.oecd.org/health/ caring-for-quality-in-health-9789264267787-en.htm

8. Dias CF, Lima SB, Fernandes GA. Scientific production on the instruments of assessment of the quality of nursing care: Bibliometric Study. Saúde (Santa Maria). 2018;44(2):1-12. DOI: http:// dx.doi.org/10.5902/2236583433477

9. Dorigan GH, Guirardello EB. Translation and cross-cultural adaptation of the Newcastle satisfaction with nursing scales into the Brazilian culture. Rev Esc Enferm USP. 2013;47(3):562-68. DOI: 10.1590/S0080-623420130000300006

10. Dempsey C, Assi MJ. The impact of nurse engagement on quality, safety, and the experience of care: what nurse leaders should know. Nurs Adm Quarterly. 2018;42(3):278-83. DOI: http:// dx.doi.org/10.1097/NAQ.0000000000000305

11. Yilmaz AT, Demirsoy N, Martins MM. Validity and Reliability Testing of the Turkish Version of the "Scale of Perception of Nursing Activities That Contribute to Nursing Care Quality (EPAECQC)". Int J Caring Sci [Internet]. 2018 [cited 2020 Mar 3];11(3):1340-51. Available from: https://internationaljournalofcaringsciences.org/ docs/2.\%20Demirsoy.pdf

12. Ribeiro OM, Martins MM, Tronchin DM. Nursing care quality: a study carried out in Portuguese hospitals. Rev Enf Ref. 2017;4(14):89-100. DOI: http://dx.doi.org/10.12707/RIV16086

13. Ryan C, Powlesland J, Phillips C, Raszewski R, Johnson A, Banks-Enorense K, et al. Nurses' Perceptions of Quality Care. J Nurs Care Qual. 2016;32(2):180-5. DOI: http://dx.doi.org/10.1097/ NCQ.0000000000000211

14. Caldana G, Hirotani LC, Gabriel CS, Silva LGC, Bernardes A. Outcome assessment tools for quality improvement programs: integrative review. Rev baiana enferm. 2018;32:e22292. DOI: http://dx.doi. org/10.18471/rbe.v32.22292

15. Martins MM, Gonçalves MN, Ribeiro OM, Tronchin DM. Quality of nursing care: instrument development and validation. Rev Bras Enferm. 2016;69(5):864-70. DOI: http://dx.doi. org/10.1590/0034-7167-2015-0151

16. Maroco J. Análise Estatística com o SPSS Statistics. Pero Pinheiro: Report Number; 2018.

17. Maroco J. Análise de Equações Estruturais Fundamentos Teóricos, Software e Aplicações. $2^{\text {nd }}$ ed. Pero Pinheiro: Report Number; 2014.

18. Cunha CM, Almeida Neto OP, Stackfleth R. Main psychometric evaluation methods of measuring instruments reliability. Rev Aten Saúde. 2016;14(47):75-83. DOI: https://doi.org/10.13037/ ras.vol14n 47.3391

19. Ribeiro OMPL, Martins MMFPS, Sousa PAF, Trindade LL, Forte ECN, Silva JMAV. Quality of nursing care: contributions from expert nurses in medical-surgical nursing. Rev Rene. 2020;21:e43167. DOI: https://doi.org/10.15253/21756783.20202143167

20. Esperidião MA, Viera-da-Silva LM. The satisfaction of the user in health services evaluation: essay on the imposition of problems. Saúde debate. 2018;42(spe2):331-40. DOI: https://doi. org/10.1590/0103-11042018s223

21. Protasio APL, Gomes LB, Machado LS, Valença AMG. User satisfaction with primary health care by region in Brazil: $1^{\text {st }}$ cycle of external evaluation from PMAQ-AB. Ciênc saúde 
Olga Maria Pimenta Lopes Ribeiro, Maria Manuela Ferreira Pereira da Silva Martins, Daisy Maria Rizatto Tronchin, Paulo João Figueiredo Cabral Teles, Letícia de Lima Trindade, João Miguel Almeida Ventura da Silva

coletiva. 2017;22(6):1829-44. DOI: https://doi.org/ 10.1590/1413-81232017226.26472015

22. Freire RM, Lumini MJ, Martins MM, Martins T, Peres HHC. Taking a look to promoting health and complications' prevention: differences by context. Rev Latino-Am Enfermagem. 2016;24:e2749. DOI: http://dx.doi.org/10.1590/1518-8345.0860.2749
23. Souza AC, Alexandre NM, Guirardello ED. Psychometric properties in instruments evaluation of reliability and validity. Epidemiol Serv Saúde. 2017;26(3):649-59. DOI: https://doi.org/10.5123/ S1679-49742017000300022

Recebido: 19 de abril de 2020

Aprovado: 28 de maio de 2020

Publicado: 14 de julho de 2020

A Revista Baiana de Enfermagem utiliza a Licença Creative Commons - Atribuição-NãoComercial 4.0 Internacional. https://creativecommons.org/licenses/by-nc/4.0/

Este artigo é de acesso aberto distribuído sob os termos da Licença Creative Commons (CC BY-NC).

Esta licença permite que outros remixem, adaptem e criem a partir do seu trabalho para fins não comerciais. Embora os novos trabalhos tenham de lhe atribuir o devido crédito e não possam ser usados para fins comerciais, os usuários não têm de licenciar esses trabalhos derivados sob os mesmos termos. 